\title{
Ultra-thin films for plasmonics: a technology overview
}

\author{
Malureanu, Radu; Lavrinenko, Andrei
}

Published in:

Nanotechnology Reviews

Link to article, DOI:

10.1515/ntrev-2015-0021

Publication date:

2015

Document Version

Publisher's PDF, also known as Version of record

Link back to DTU Orbit

Citation (APA):

Malureanu, R., \& Lavrinenko, A. (2015). Ultra-thin films for plasmonics: a technology overview. Nanotechnology Reviews, 4(3), 259-275. https://doi.org/10.1515/ntrev-2015-0021

\section{General rights}

Copyright and moral rights for the publications made accessible in the public portal are retained by the authors and/or other copyright owners and it is a condition of accessing publications that users recognise and abide by the legal requirements associated with these rights.

- Users may download and print one copy of any publication from the public portal for the purpose of private study or research.

- You may not further distribute the material or use it for any profit-making activity or commercial gain

- You may freely distribute the URL identifying the publication in the public portal

If you believe that this document breaches copyright please contact us providing details, and we will remove access to the work immediately and investigate your claim. 


\section{Review}

\section{Radu Malureanu* and Andrei Lavrinenko \\ Ultra-thin films for plasmonics: a technology overview}

DOI 10.1515/ntrev-2015-0021

Received March 17, 2015; accepted May 8, 2015

\begin{abstract}
Ultra-thin films with low surface roughness that support surface plasmon-polaritons in the infra-red and visible ranges are needed in order to improve the performance of devices based on the manipulation of plasmon propagation. Increasing amount of efforts is made in order not only to improve the quality of the deposited layers but also to diminish their thickness and to find new materials that could be used in this field. In this review, we consider various thin films used in the field of plasmonics and metamaterials in the visible and IR range. We focus our presentation on technological issues of their deposition and reported characterization of film plasmonic performance.
\end{abstract}

Keywords: plasmonics; ultra-thin films; VIS/IR range.

\section{Introduction}

Plasmonics currently lives through the revival period after its first successful appearance in the 1980s with findings summarized in Raether's monograph [1]. In his broad overview of perspective developments in paradigm of CMOS compatible silicon photonics, Richard Soref mentions plasmon optics or plasmonics as one of the photonics key instrument to reach extreme light localization, deep subwavelength resolution and enhanced light emission [2]. Such properties stem from light-free electron coupling occurring at metal-dielectric interfaces. The carriers of such interaction are surface plasmons, which occur in two basic configurations: localized surface plasmons with

\footnotetext{
*Corresponding author: Radu Malureanu, Department of Photonics Engineering, Technical University of Denmark, Oersteds plads, bldg. 343, 2800, Kgs. Lyngby, Denmark, e-mail: rmal@fotonik.dtu.dk Andrei Lavrinenko: Department of Photonics Engineering, Technical University of Denmark, Oersteds plads, bldg. 343, 2800, Kgs. Lyngby, Denmark
}

deep analogy in mechanical oscillations and surface plasmon-polaritons (SPPs) or propagating surface plasmons analogous to mechanical waves [3]. In this review, we will focus on fabrication issues mostly related to SPPs propagation. By coupling to the ensemble of free electrons, light can be effectively confined and, as a result, guided. For such alliance of characteristic temporal parameters in correspondence with optical frequencies and spatial parameters of electronic footprints, plasmonics has been intensively praised for bridging photonics and electronics. A special direction of plasmonic circuitry has been successfully propelled for a while, see, for example [4].

However, very fast the main trade-off of plasmonics was built off: losses vs. confinement [5]. The more plasmons are confined to the interface, the bigger are losses conventionally associated with the process of free electron transport or displacement in metals followed by electronelectron and electron-phonon scattering. This SPPs loss channel, which exists even in the ideal case of perfectly smooth metal layer interfaces is expectedly appended by intensive scattering of surface plasmons on possible surface/bulk imperfections and defects including the ones of extremely small sizes (of a nanometer scale, like metal grains) as a direct consequence of big wavevectors of SPPs in comparison with light waves of the same frequency [6].

Thus, advancing in terms of plasmonic circuitry inevitably brings a challenge of fabrication as flat as possible interfaces. This strict constrain clashes with another requirement, often imposed on fabrication processes - to deposit ultra-thin metal layers with typical thicknesses below $20 \mathrm{~nm}$. Such requirement is erected both in plasmonics and metamaterials. In the former, the losses of socalled long-range SPPs considered together with hybrid plasmonic-photonic modes as a workhorse in guiding [7] are inversely dependent on the guiding (metal) layer thickness. In the latter case, a new trend in hyperbolic metamaterials claims a metal-dielectric multilayer as the basic device possessing hyperbola-like dispersion bands [8]. In employing such device as a hyperlens, the quality of the lens, e.g. its ability to image subwavelength elements with high contrast is determined by the metal layer thickness 
and smoothness [9]. Together, both requirements impose a very challenging message for current fabrication technologies implying almost reaching the technological limits of depositing thin layers. As such, new advances in the deposition of such layers have to be made.

Conventionally plasmonic materials are associated with metals. This is because the coupling to free electrons anticipates their presence in excess in a material. From the phenomenological point of view, it means that standard solutions of Maxwell's equations for surface waves require different signs of material constants, e.g. permittivity functions, in media in contact. Accordingly to the classical model of the free electron gas - the permittivity is represented by the Drude formula, and the real part of the permittivity is negative below plasma frequency $\omega_{\mathrm{p}}$. For typical frequencies of the visible and near infra-red (IR) ranges, such noble metals like $\mathrm{Au}, \mathrm{Ag}$, and $\mathrm{Al}$ are the best candidates having $\omega_{\mathrm{p}}$ further away in the ultraviolet. Sometimes, $\mathrm{Cu}$ is considered as well. However, despite low losses, their universality in plasmonic applications has been seriously challenged initiating a quest for alternative plasmonic materials $[10,11]$. Not the last role in stimulating the search of alternative solutions plays fabrications issues: requirements for ultra-thin uniform layers as we specified above and CMOS compatibility. Silver and gold as the best so far plasmonic materials in terms of losses in the visible and near-IR possess the high surface energy leading to granulation for very small film thicknesses and challenges for low-loss adhesion layers. Also, these metals are beyond the list of materials accepted in semiconductor growth processes. $\mathrm{Al}$ and $\mathrm{Cu}$ are CMOS compatible, but in terms of losses are considered worse than gold and especially silver.

The list of alternative plasmonic materials is really vast now, see, e.g. [7] and references therein. Some of them are promising and draw a lot attention, like CMOScompatible transition metal nitrides (TiN, TaN, etc.) $[12,13]$ or II-VI semiconductors oxides (InTiO, Al:ZnO, Ga:ZnO, etc.) $[14,15]$. Others seem to be appropriate substitution of metals in mid- and far-IR or even in the terahertz range, where metallic properties of noble metal become too strong, and their behavior resembles more perfect conductors ousting truly plasmonic features. Here, typically heavily doped semiconductors $[16,17]$ and so-called two-dimensional atom-thick materials like graphene $[18,19]$ are considered.

Such variety of materials definitely brings a challenge in formulating common criteria for evaluation of their plasmonic performance and processing challenges. Aiming the fabrication issues in reaching ultrathin uniform smooth layers, we put forward material properties and fabrication peculiarities. The main parameters to be considered for improving the functionality are roughness/uniformity, range of available or demonstrated thicknesses and requirements for an adhesion or wetting layer. As for optical properties, if possible SPP loss of characterization will be given, however, in many cases, data are missing.

The structure of the review is the following. In the second section, we briefly remind deposition/growing techniques and methods for characterization film properties. The overview of advances in depositing thin metal films on various surfaces follows. Techniques for depositing gold and silver come first. Then, we describe the deposition of $\mathrm{Al}, \mathrm{Cu}, \mathrm{Cr}, \mathrm{Ni}, \mathrm{Ti}, \mathrm{Fe}$, and alkali metals pointing on their possible employment in plasmonic problems. The next section deals with transparent conductive oxides and nitrides, the main ones being ITO, AZO, and TiN. Section IV handles highly doped III-V semiconductors. Twodimensional materials apt for plasmonic applications are considered in Section V. Prospective view on fabrication advances and conclusions are placed in the last section.

\section{Deposition/growing and characterization techniques}

In the following, we shortly overview typical fabrication techniques for depositing/growing thin plasmonic layers and approaches in characterizing the obtained structures. As the aim of the review is not a full description of the techniques, for this, a book on the subject would be more appropriate; these descriptions are to be understood as only presenting the principles of the techniques and not providing extended details of their complete capabilities.

\subsection{Deposition/growing techniques}

Generally, the deposition techniques can be divided into several categories. We will first present an overview of physical vapor deposition (PVD) techniques, then followed by a description of the chemical vapor deposition (CVD), and at the end, the techniques that are in some extent a combination of the chemical and vapor phase techniques or are based on chemical synthesis.

For metal deposition, the most used technique is evaporation $[20,21]$. Its principle is to heat the metal to be deposited in a controlled manner. So atoms will evaporate from a target at a prescribed rate and eventually rest on the desired substrate. Depending on the equipment used, 
there are two main types of evaporation techniques: electron beam (e-beam) evaporation where the metal target is heated with the help of a beam of electrons directed at the metal surface, and thermal evaporation, where metal is heated using the Joule effect. It should be noted that e-beam evaporation may be used also for depositing dielectrics.

Another deposition technique, mainly used for metals but that also could be used for dielectrics and semiconductors, is sputtering $[20,21]$. In the process, a target material is bombarded with high-energy particles such that the atoms are knocked out from the target and reach the substrate. The most common used sputtering technique is magnetron sputtering. In this case, due to the strong electric and magnetic fields generated inside the magnetron, plasma is formed and confined close to the target material. When depositing dielectrics, there is a risk of charge accumulation, thus, generally a high-frequency variation of the anode-cathode bias is applied. In this case, sputtering is generally referred to as $\mathrm{RF}$ (radio frequency) sputtering.

While there exist many variations of sputtering techniques, for example, ion-beam sputtering, reactive sputtering, ion-assisted deposition, high-target utilization sputtering, and high-power impulse magnetron sputtering, unless otherwise specified, we will refer to magnetron sputtering throughout the article.

The next PVD technique is molecular beam epitaxy (MBE) [20, 21]. In this case, a target or targets are slowly heated to obtain sublimation of the desired material that will reach the substrate and be deposited there monoatomically. Owing to the low deposition rate, there will be an epitaxial growth of the layers, thus, yielding a much smoother film. It should also be noted that, although it is a physical vapor deposition technique, when using more than one target, a chemical reaction on the substrate surface might take place, thus, resulting in forming of, for example, GaAs layers from separate Ga and As targets.

Pulsed laser deposition (PLD) is also a PVD technique $[20,21]$. A high-power laser pulse is focused on the target material. The vaporized material then creates a plasma plume that will eventually reach the substrate and, thus, deposit material on it. Although the setup is relatively simple, due to the presence of various species (from electrons and ions to atoms and clusters) in the plasma plume, controlling the deposition characteristics is not straightforward.

Apart from the PVD techniques, there are also several CVD ones.

The most common CVD method is plasma-enhanced chemical vapor deposition (PECVD) [20, 21]. This technique is based on mediating a specific chemical reaction in a plasma environment, thus, to deposit the desired composition on a prescribed substrate. It generally involves a high temperature and relatively complex chemistry, but it is the basic technique for graphene deposition, for example.

Another type of CVD is atomic layer deposition (ALD) technique [20, 21]. The main difference between the ALD and PECVD techniques is the chemical reaction dynamics. Thus, the ALD reaction is self-limited by the reaction dynamics and, as such, in one cycle a monolayer of a specific material is obtained.

Metal-organic vapor phase epitaxy (MOVPE) [20, 21] is a deposition technique that is based on a complex combination of vapor phase deposition and chemical reactions. As it is simultaneously chemical and physical vapor depositions, MOVPE is also called MOCVD. While in the MBE case the chemical reaction may or may not be used during the deposition, in the MOVPE, it is a necessity. The reaction between the species present in a machine creates the condition for crystals to grow, thus, enabling obtaining the desired material.

Other deposition approaches involve the use of chemical reactions in solution with a reduction of a smooth layer of material on the substrate. The electroless technique for depositing thin Ag layers [22, 23], and solution-dipping processes for $\mathrm{Al}$ [24] are examples of these techniques. They involve the reaction of at least two species in solution, and the desired material is formed and deposited on the substrate, while the by-products remain in solution.

\subsection{Characterization techniques}

The characterization techniques can be split in two main categories. On one side, the topological and composition characterization will describe the layer quality in terms of roughness, completeness, uniformity, thickness composition, etc. On the other side, the functional characterization deals with the description of the deposited layers in terms of their response to incoming radiation, electrical or chemical stimuli, etc. First, we will review the topological characterization techniques.

The main methods for determining the topology of the deposited layers are scanning electron microscopy (SEM) and atomic force microscopy (AFM). They are generally used in conjunction with each other as they produce complementary information.

The SEM is based on the interaction of a focused electron beam with a surface of interest that is scanned by the beam [25]. The emitted secondary electrons are captured by a detector, and a pixel-by-pixel image is created. 
Although the obtained image is generally very similar to a topographical image of the surface, there is no direct correlation between them. As the SEM image represents the emitted electrons, it should be considered as an interpreted image. Peaks and valleys in the image are not necessary topographical ones, and the information about the axis perpendicular to the image plane is, at most, qualitative. Still, it will show, e.g. the areas where there is no coverage with the desired layer, number of particles present on the surface, etc. Sometimes, SEM techniques are combined with electron dispersion X-ray spectroscopy (EDX) technique. Using EDX, the chemical composition of the sample can be analyzed quantitatively, thus, allowing for the determination of its chemical purity and composition.

The AFM imaging uses a completely different procedure to obtain information about the structure. In this case, a sharp tip of a few nanometer curvature radius is placed in the vicinity or on top of the surface of interest [26]. While scanning the surface, the tip deflection is measured using a laser spot, and as such, quantitative information about the surface topography is obtained. However, the information obtained will not give any indication of the nature of the substrate, thus, it is hard to differentiate between areas, where the desired material is deposited and where only the substrate is present. More advanced AFM includes also the possibility of recognizing chemical species based on the interaction between the tip and the substrate.

Transmission electron microscopy (TEM) is a technique relatively similar to SEM, but in this case, the collected electrons are transmitted through the sample rather than reflected from the sample surface [27]. The main limitation of the TEM is the thickness of the sample. Thus, in order for the electrons to pass through the sample, it needs to be just several tens of nanometers thick. Various imaging postprocessing modes exist, which can provide detailed information about the sample ranging from topographical imaging to lattice parameters and chemical composition.

Scanning tunnelling microscopy (STM) is a technique where a sharp tip is scanned on top of a specimen [28]. Different from AFM, there is a bias applied between the tip and the substrate. The current that tunnels through is a function of the local density of states. As the resolution obtained is generally below $0.1 \mathrm{~nm}$ laterally and $10 \mathrm{pm}$ vertically, STM can provide information about the atomic structure of the scanned sample.

Auger spectroscopy technique is based on exciting an atom by an external field [29]. If the external field is strong enough, a core electron may be expulsed leaving a hole. Such an unstable state will lead to the decaying of an electron from the outer shell. The resulting obtained radiation may lead to a second electron being expelled from the atom. This second electron is a so-called Auger electron, and by measuring its energy, one can determine the atomic species and, thus, the chemical composition of a specimen. Although quite complex, this technique is now routinely used for determining the elemental composition of surfaces.

X-ray photoelectron spectroscopy (XPS) is another technique for determining the chemical composition of a surface [29]. In this case, the specimen bombarded by an X-ray beam will emit electrons, and by analyzing the number of the electrons and their energy, the chemical composition of the surface can be determined.

Reflection high-energy electron diffraction (RHEED) [30] is a technique, where an electron beam is incident at a glazing angle on the surface of interest. The diffracted electrons will interfere and, as such, create patterns that will provide information about the crystal structure and lattice parameters of the specimens' surface.

From the functional point of view, there are a few techniques that are mainly used. The four-point probe technique is based on accurately placing four probes at predetermined distances on a substrate. Using two of the probes for providing a potential difference and measuring the current with the other two, one can accurately measure the specimen conductivity. By corroborating these measurements with the theoretically predicted ones, the electrical characteristics of the deposited layer can be estimated.

In order to characterize the behavior of surface plasmons-polaritons on thin metal films, there are several ways possible. Scanning near-field optical microscopy (SNOM) is a technique where a SPP field is measured using an AFM-like probe [31]. When the SPP is propagating along a surface, its field will decay exponentially away from the metal-air interface. By placing a probe in near vicinity of the surface, this field can be captured and measured, thus, providing information about the SPP behavior. In the same time, due to its AFM characteristics, the field distribution can be correlated to the topographical one.

Another option is to use the Kretschmann or Otto setup. As the wavevector of surface plasmons-polaritons is bigger than the one of light waves in free space, in order to excite a SPP, one has to use a high-index prism. Both Otto and Kretschmann configurations employ such prism, the main difference between the two being the type of the separating layer between the interface supporting the surface plasmon-polariton and the prism. In the Otto case, this layer is of low index dielectric, and the SPP propagates at the interface between this spacing layer and the 
metal, or metal-like one. In the Kretschmann case, the metal or metal-like layer is in close contact with the prism and is actually the separating layer. The SPP propagates at the interface between this layer and free space (generally) beneath the layer. In both cases, by measuring the reflected spectra of a beam incident on the prism, one can determine the existence of the plasmon certified by dips in reflection spectra.

A third option for characterization of the layers' quality is to fabricate a waveguide and measure SPP transmission through it. By considering several waveguides of various dimensions, the propagation length of the plasmon can be deduced.

The last technique presented here is to measure the reflection and transmission spectra of a layer and, from here, determine its optical characteristics, and with more advanced measurements, the dispersion diagram of the material measured. Although reflection-transmission spectroscopy is a very powerful technique that provides important information about the layer quality, it cannot give direct evidence about the presence of an SPP at the interface. A more developed technique, but based on the same principle is ellipsometry. In this case, the reflectioninduced ellipticity of a circularly polarized beam can provide information about optical and material properties of the film.

Although there are several other techniques, like leakage microscopy technique or electron energy loss spectroscopy, the above-mentioned ones are the most used, thus, we limited ourselves to their brief description.

\section{Metallic films}

By far, the most used materials for plasmon propagation in the visible and IR ranges are metals. Owing to their high electron mobility, they hold the promise of having the lowest losses at these frequencies. Out of the multitude of possibilities, gold, silver, and aluminum are the ones that are mainly used. Although the alkali metals are theoretically better due to their even higher electron mobility and, thus, lower losses, their notorious chemical and mechanical instability makes them impractical to work with. There have been only very few attempts where plasmons in these metals were characterized [32].

\subsection{Gold thin films}

Gold is the plasmonic material mostly used nowadays. Its advantages are high chemical stability and electron mobility. It can be deposited in various ways, from electron-beam evaporation to sputtering, but due to its chemical inertness, it cannot be deposited using precursors, as in atomic layer deposition machines.

Surface plasmons were theoretically predicted by Ritchie in 1957 [33], but the first experimental proof was done by Kretschmann in 1971 [34]. The method he developed is still used today as one of the methods for exciting this kind of waves. In his work, he used gold evaporated directly on the surface of a prism, thus, being able to excite the desired plasmons. Gold was used as the material of choice for exciting plasmons since then. Its biocompatibility helped in developing various techniques where surface plasmon-polaritons excited in gold films were used for enhancing Raman spectra [35] or measuring the growth of thin organic films [36]. But in the same time, the existence of surface plasmon-polaritons allowed for better characterization of the optical properties of gold [37].

In the last 15 years, the plasmonic waveguides came to prominence due to their possibility of confining light at a scale much smaller than its wavelength. This allows, in theory, for highly compact interconnects between various optical devices. On the other hand, due to the pronounced losses in such waveguides, the characteristic propagation distance of surface plasmon-polaritons is typically very small. Thus, a compromise between the light confinement and losses must be found.

In order to avoid unnecessary losses in metal waveguides, surface roughness and the film's uniformity should be as perfect as possible. This challenged the depositing techniques to evolve in order to produce ultrasmooth metallic layers. An additional requirement on the layer thickness also targets to make them ultra-thin.

The first impediment in depositing smooth thin gold films is the high percolation threshold. The combination of high atom mobility and surface tensions effect leads to fractal-like cluster to be formed when depositing gold [38]. Owing to these effects, the thinnest complete gold layer that can be deposited on silicon or silica is in the order of $20 \mathrm{~nm}$. In the same time, apart from the relatively large minimal thickness, the obtained layer is quite rough, with measured roughness up to $4 \mathrm{~nm}$ [39].

Various studies were made in order to understand and counterbalance this effect. The accent was put on in situ measurements that can allow for better characterization of the growth dynamics on Au layers. X-ray reflectivity and scanning tunnelling microscopy are the ones mainly used [40, 41] but in situ Auger electron spectroscopy [42] and ex situ pull testing [43] were also reported among others.

To reduce this high percolation threshold, the most straightforward technique is to deposit, prior to the gold 

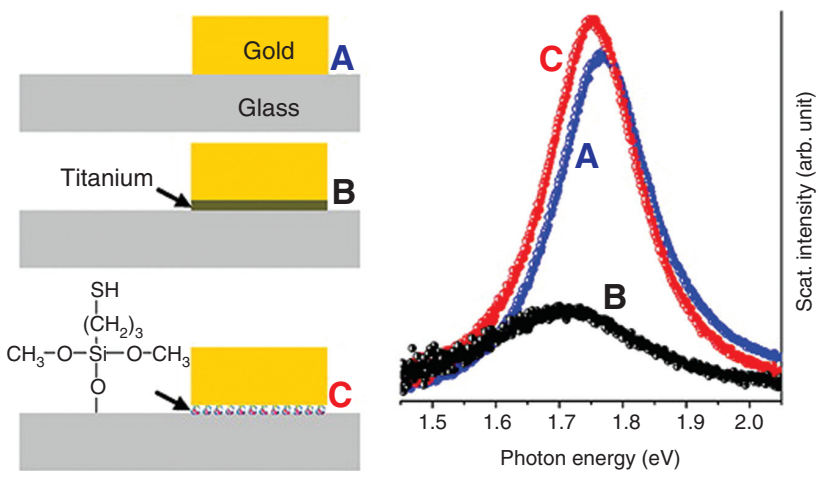

Figure 1: Influence of the adhesion layer on the scattering spectra of single Au nanorods. (A) bare gold layer; (B) gold layer deposited on the Ti adhesion layer; (C) gold layer on top of the mercaptopropyl functionalized surface. Reproduced with permission from [46].

layer, an adhesion layer. This way, the surface characteristics change, and gold can be deposited as thin complete layers. The downside of this technique is the introduction of an extra layer in the system, thus, potentially changing the characteristics of the structure.

Two of the most used adhesion layers are chromium $[44,45]$ and titanium. Although they allow for a smoother and thinner waveguide, neither of these metals has the optical characteristics superior to gold; thus, the plasmon behavior can worsen significantly. Their use is extremely important for thin gold layer deposition, but in the same time, their influence on the plasmon characteristics increases. For example, even keeping the titanium adhesion layer to a minimum of ca. $2 \mathrm{~nm}$, for a system where the gold layer is $17 \mathrm{~nm}$, the contribution of the adhesion layer significantly deteriorates the overall performance of a golden nanorod [46] (see the scattering resonances in Figure 1). Trying to reach even lower thicknesses will increase the ratio between the gold and titanium contributions, thus, the waveguide or particle characteristics will be even worse. A much less used metallic adhesion layer, which promises forming of a very smooth $\mathrm{Au}$ layer on sapphire is $\mathrm{Nb}$ [47]. In this case, the $\mathrm{Au}$ layer tends to be monocrystalline even at thicknesses down to $10 \mathrm{~nm}$.

Polymers, on the other hand, may be used as an adhesion layer, although their influence was not thoroughly investigated. Ormocomp shows the possibility of allowing for 12-nm-thick layers to be created [48], but a systematic study was not, to the best of our knowledge, made. Another polymer layer used as a seed layer is Cyclotene (also known as BCB) [48]. Leosson et al. also show the possibility to reach thin layers with thicknesses of about $10 \mathrm{~nm}$ (Figure 2). Owing to the lack of comprehensive study on this topic, we cannot say anything about result reproducibility or layer characteristics.

Preliminary experiments also show that extremely thin $\mathrm{Au}$ films may be deposited on conductive oxides [49]. Although there are no detailed studies, layers as thin as $2.4 \mathrm{~nm}$ of Au may be sandwiched between two ITO layers using pulsed laser deposition methods.

Amino- and mercapto-silanes [46, 50-52] were investigated as adhesion layers. Both these organosilane compounds exhibit great promise for obtaining ultra-thin gold layers with deposition of metal performed by conventional electron beam or thermal evaporation techniques. Authors report deposition of gold on a silicon wafer pretreated with the amino-silane compound
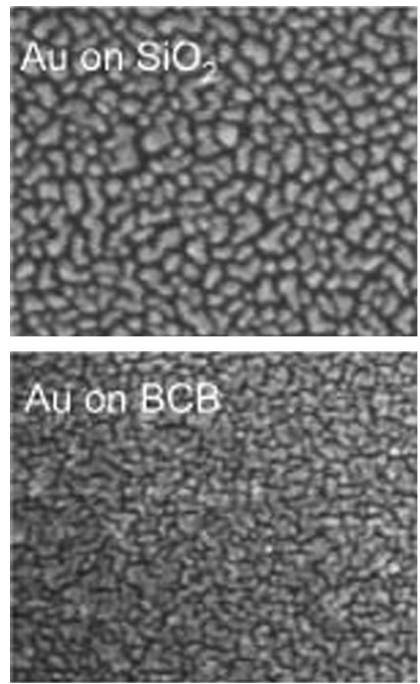
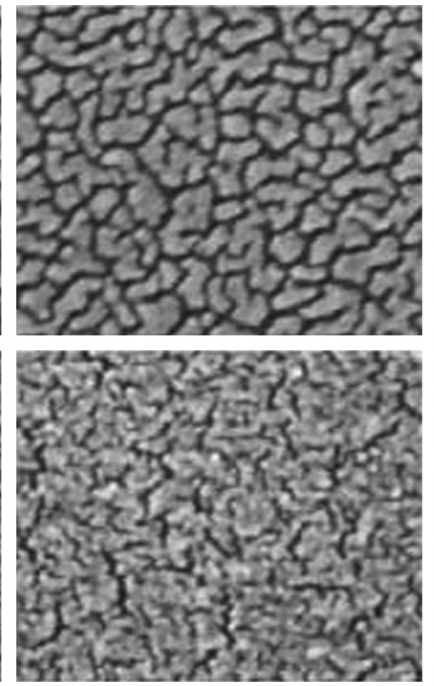
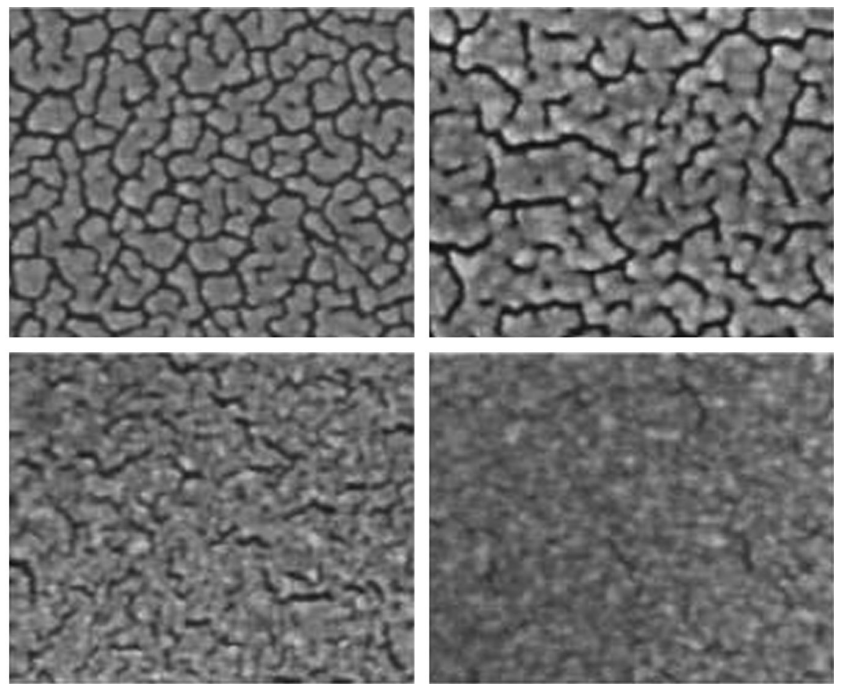

Figure 2: Ultrathin (3, 4.2, 5.4 and $7.2 \mathrm{~nm}$ respectively) Au layers on pure silica (upper row) and on BCB polymer (lower row). Reproduced with permission from [48]. 
reaching a thickness of as thin as $6 \mathrm{~nm}$ [51] and on fused silica wafers pretreated with mercapto-silane with the mass equivalent thicknesses of $5.4 \mathrm{~nm}$ [52]. Optical characterization of such metal layers showed that the obtained gold layer has properties very close to the one of pure bulk gold $[46,52]$.

The sputtering techniques are also commonly used for depositing Au layers. Thus, using optimized sputtering, gold was deposited on silica/silicon with a surface coverage of ca. $90 \%$ and an approximate thickness of $7.6 \pm 0.4 \mathrm{~nm}$ [52]. Although the surface coverage below $100 \%$ witnesses the presence of holes in the layer, having an almost complete layer at these thicknesses on silica substrates is an achievement in itself.

In the same time, it was reported that atomically flat $\mathrm{Au}$ layers can be obtained on special substrates like mica [53] and $\mathrm{LiF}$ [54] using sputtering techniques. The obtained gold layers were very smooth, the RMS in the latter case was $0.2 \mathrm{~nm}$ (but the thickness was $80 \mathrm{~nm}$ ). However, the substrates used either have too high losses for the visible or infrared ranges, as in the case of mica, or impractical (due to its toxicity) to be used in mass production as in the $\mathrm{LiF}$ case.

A different approach involves evaporating $\mathrm{Au}$ on a mica substrate and then peeling it off from the initial substrate and attaching to a silica one $[55,56]$. This approach allows for extremely flat layers (RMS $=0.275 \mathrm{~nm}$ [55]) to be created (Figure 3), but it does not preclude the use of

A
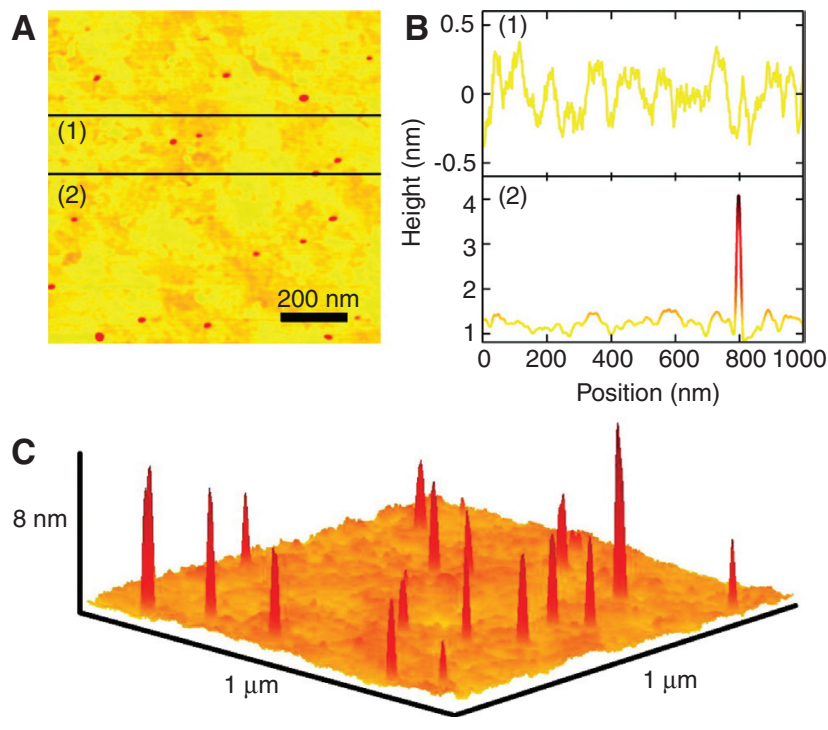

Figure 3: Ultrasmooth gold layers obtained by stripping from mica substrates. (A) CdSe quantum dots on a gold layer; (B) height profiles of a flat gold layer and a layer with a quantum dot; (C) three-dimensional AFM image of quantum dots on the gold layer. Reproduced with permission from [55]. metallic adhesion layers in order to insure good adhesion between the silica superstrate and the gold.

Another attempt to obtain smooth layers of $\mathrm{Au}$ on silica involves thermal annealing of a thin gold coating in nitrogen atmosphere [57]. High temperature (up to $814^{\circ} \mathrm{C}$ ) allows for obtaining monocrystalline $\mathrm{Au}$ embedded in the Si lattice. Lower annealing temperatures $\left(350^{\circ} \mathrm{C}\right.$ and $626^{\circ} \mathrm{C}$ ) lead to nanoislands randomly dispersed on the surface without epitaxial relationship. Thus, no complete layers were formed impeding their implementation in plasmonic devices.

Recently, using chemical synthesis, monocrystalline gold flakes were controllably grown [58]. These flakes exhibit the ultimate smoothness but have several disadvantages. First of all, they are still relatively thick and their thickness is dependent on the size, thus, for bigger structures, they need to be thicker. Second, they are grown in solution, and when deposited on a substrate, their position is difficult to control accurately. Owing to this, the possibility for this method to be used in largescale production is limited. A short overview of thin $\mathrm{Au}$ film parameters is shown in Table 1.

We should also mention that there were few attempts to deposit gold using CVD methods [59, 60]. In these cases, the gold loading is low, and no any complete layer was formed. To our knowledge, there are no CVD processes in which $\mathrm{Au}$ can be reliably deposited in thin films.

\subsection{Silver thin films}

Silver is another metal with huge potential in the plasmonics and metamaterials research field. Although it has theoretically lower losses than gold [10], it is less utilized due to its notorious chemical instability [61]. While gold is stable once deposited, silver, if not capped with a protection layer, oxidizes fast permanently degrading in plasmonic properties.

Most of the techniques used for improving gold deposition, optimizing the adhesion layers and the

Table 1: Thin Au film parameters on different substrates and using various adhesion layers.

\begin{tabular}{lllll}
\hline Thickness & RMS & Adhesion layer & Substrate & Ref. \\
\hline $10 \mathrm{~nm}$ & $0.2-0.3 \mathrm{~nm}$ & $\mathrm{Nb}$ & C-cut sapphire & {$[47]$} \\
$4-15 \mathrm{~nm}$ & $1 \mathrm{~nm}$ & & BCB, Ormocer & {$[48]$} \\
$2.4 \mathrm{~nm}$ & & & ITO & {$[49]$} \\
$6-10 \mathrm{~nm}$ & $0.26 \mathrm{~nm}$ & Organosilane & Silicon & {$[51]$} \\
$5.4-15 \mathrm{~nm}$ & $0.6 \mathrm{~nm}$ & Mercatopsilane & Fused silica & {$[52]$} \\
$80 \mathrm{~nm}$ & $0.2 \mathrm{~nm}$ & & LiF & {$[54]$} \\
$150 \mathrm{~nm}$ & $0.275 \mathrm{~nm}$ & $\mathrm{Cr}$ & Mica & {$[55]$} \\
\hline
\end{tabular}


deposition parameters as well as using different substrates, can also be employed for obtaining thin silver films. But, due to its reactivity, thin silver layers can be obtained with the bigger nomenclature of methods including electroless techniques [22, 23, 62] and CVD methods [63].

Epitaxial growth of silver films is, to our knowledge, the most advanced method to obtain thin smooth layers [64]. Thus, using a freshly cleaved GaAs (110) substrate and combination of a low deposition rate at low temperature and annealing at room temperature $[65,66]$ one can obtain atomically flat ultrathin silver films (Figure 4). This method also allows the determining of a critical thickness below which silver cannot be deposited as a layer: $15 \AA$. Although the obtained film has the ultimate flatness with a maximum height difference below $0.5 \AA$, it has the major disadvantage of not being a continuous layer. During the annealing process, the silver atoms rearrange and form areas of extreme smoothness but also voids where no silver is present. Still, this technique is invaluable to obtain smooth layers, and it was further developed for silicon substrates $[67,68]$ showing major improvements of the film plasmonic characteristics.

Thin silver films can be obtained also using RF magnetron sputtering [69]. In this case, the film is not monocrystalline, but rather nanocrystalline, thus having roughness in the order of $0.18 \mathrm{~nm}$ for a $14 \mathrm{~nm}$-thick layer of silver. The roughness increases to $2.5 \mathrm{~nm}$ with the growth of the layer thickness as a direct consequence of the grain size enlargement. Still, it provides an extremely smooth layer that may be used for plasmonic devices. Another big advantage of this technique is the use of glass as a substrate, thus, making samples readily available for visible and IR plasmonics. Using DC magnetron sputtering [70], one can obtain monocrystalline silver layers; however,

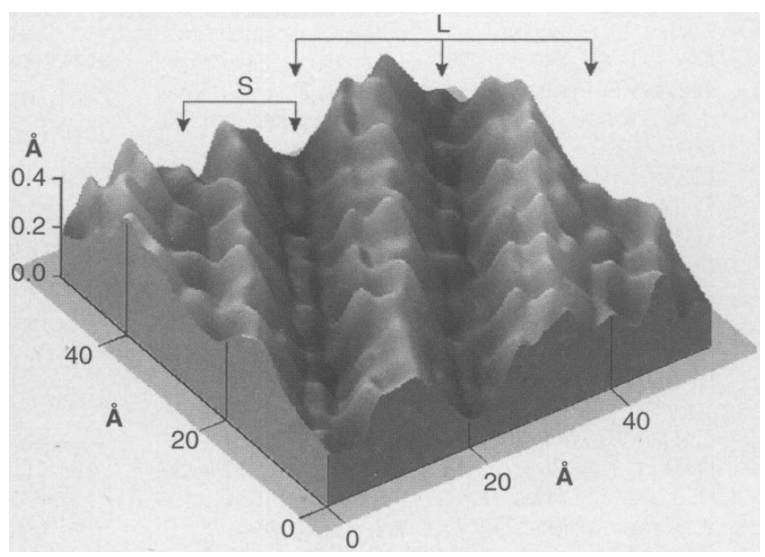

Figure 4: A 3D rendering of an STM image showing atomically smooth silver layers. Reproduced with permission from [65]. the substrate has to be lattice matched. In the reference, mica is used as the substrate, making silver films unapt for visible/IR plasmonics.

The ALD technique is also used for obtaining a thin smooth silver film [71]. This technique is extremely limited due to the narrow temperature window of a maximum $20^{\circ}$ and the difficulty in obtaining suitable precursors. Still, it allows for conformal deposition of silver on high-aspect ratio structures. The thinnest layer obtained in this case is in the order of $10-15 \mathrm{~nm}$, but it is extremely rough, an approximate roughness being above $8 \mathrm{~nm}$. However, measurements of resistivity reveal that a $40-\mathrm{nm} \mathrm{Ag}$ film deposited by ALD has resistivity $6 \mu \Omega \mathrm{cm}$ in contrast to a 40-nm CVD-produced film with resistivity $80 \mu \Omega \mathrm{cm}$.

Silver can also be deposited chemically $[22,23,62$, 72]. In this case, a reducing reaction is used to obtain thin silver films, reaching down to $10 \mathrm{~nm}$ thickness. The major disadvantage in this case is the small grain dimensions (Figure 5) and, thus, potentially numerous defects. Also, for very thin films, it is very difficult to obtain uniform coverage on large areas.

In the case where the smoothness is paramount over the optical properties, one could use Ge as an adhesion layer (Figure 6) [73, 74]. As described by Logeeswaran et al. [75], using Ge has the advantage of not only obtaining much smoother layers with up to one order of magnitude less RMS, but also increased temperature stability of such layers. Another material that can be used as an adhesion layer for silver deposition is molybdenum trioxide $\mathrm{MoO}_{3}$ [76]. As a dielectric, it brings the advantage of being utilized as an adhesion layer, reducing the losses, but still, the silver layer is not continuous. Also, up to the $0.5-\mu \mathrm{m}$ range, the $\mathrm{MoO}_{3}$ has relatively high losses [77]. Although

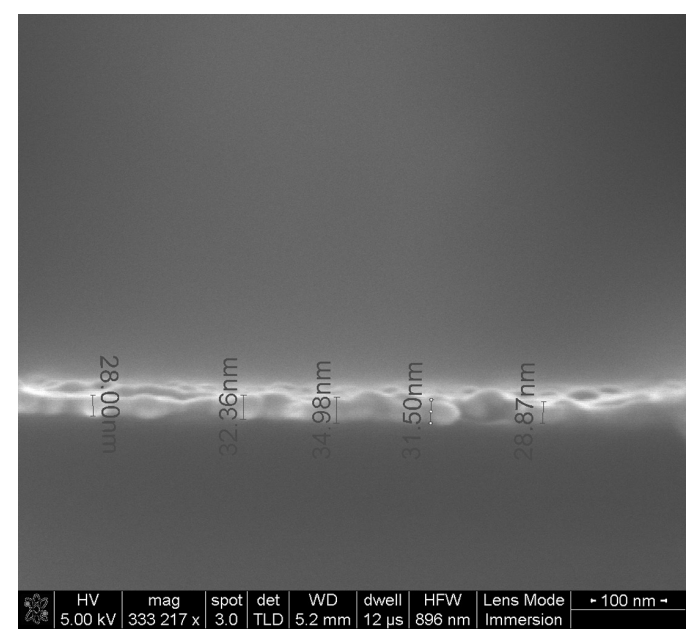

Figure 5: A 30-nm-thin Ag layer obtained using electroless techniques. Reproduced with permission from [22]. 


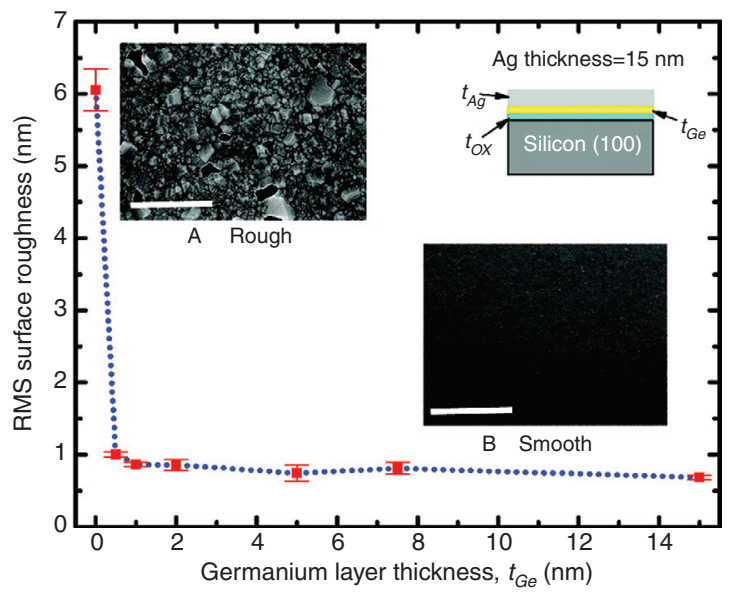

Figure 6: Variation of the RMS with the Ge seed layer thickness. Reproduced with permission from [73].

less used, aluminum-doped zinc oxide is a possible adhesion layer [78]. The authors claim Ag layer thicknesses down to $10 \mathrm{~nm}$, and as it is a sandwich-like structure, the degradation of the layer is highly reduced. $\mathrm{Cu}$ is also used as an adhesion layer for depositing Ag films [79] reaching thicknesses as low as $6 \mathrm{~nm}$.

As with gold [55], a promising technique for obtaining thin smooth silver layers consists of silver deposition on smooth substrates with poor adhesion (mica, silica, silicon) and covering them with strongly adhesive materials like epoxy. Peeling off the obtained layer from the initial substrate - so-called template stripping [80], produces a metal layer with roughness dictated by that of the initial substrates. The partial disadvantage of this method is the formation of only one working surface because another one can be very rough. Of course, it is not relevant if the thickness of a metal layer is exceeding $100 \mathrm{~nm}$.
An interesting technique for obtaining thin silver layers with reduced roughness is the co-sputtering deposition of $\mathrm{Ag}$ and $\mathrm{Al}$ [61]. The obtained films are Al-doped Ag with doping concentrations of up to $4 \%$ that allows for smooth and thermally stable layers. The presence of $\mathrm{Al}$ will definitely influence the optical characteristics [61], but its influence will probably be minimal compared to the one of, e.g. Ge wetting layer due to the better optical properties of $\mathrm{Al}$ with respect to $\mathrm{Ge}$.

Last, smooth silver films can be pressure induced [6]. In this case, the RMS is $1 \mathrm{~nm}$ or even below down to an impressive $0.1 \mathrm{~nm}$, but the resulting thickness of the $\mathrm{Ag}$ layer is not well defined. Also, the area with smooth silver is determined a posteriori, and it cannot cover the whole wafer, but only specific parts. Last, no any optical characterization of such films' appropriateness for plasmonic applications has been performed so far. An overview of Ag thin films may be found in Table 2.

\subsection{Aluminum thin films}

Aluminum as a plasmonic material has some advantages, for example, the deep UV position of the plasma frequency, high carrier concentration, and CMOS compatibility. In terms of losses, it is worse than silver and gold, but still falls in the range of reasonable materials in plasmonic applications. There are comparatively very few articles where aluminum is applied as a plasmonic metal, and as such, techniques for depositing extremely thin films have not been fully developed. Thus, an ISI Web of Knowledge search for articles that include aluminum together with the keywords plasmon or metamaterial gives a total

Table 2: Thin Ag film parameters on different substrates and using various adhesion layers.

\begin{tabular}{|c|c|c|c|c|}
\hline Thickness & RMS & Adhesion layer & Substrate & Ref. \\
\hline $100 \mathrm{~nm}$ & $1 \mathrm{~nm}$ & By pressure & $\mathrm{Si}$ & [6] \\
\hline $6 \mathrm{~nm}$ & 0.37 & Al doped & Silica/Si & {$[61]$} \\
\hline $45 \mathrm{~nm}$ epitaxial & $0.36 \mathrm{~nm}$ & & Silicon & {$[64]$} \\
\hline $50 \mathrm{~nm}$ thermal & $3.27 \mathrm{~nm}$ & & Silicon & [64] \\
\hline $1.5 \mathrm{~nm}$ & Atomically smooth & & GaAs & {$[65]$} \\
\hline $80 \mathrm{~nm}$ monocrystalline & $0.37 \mathrm{~nm}$ & & Si & [68] \\
\hline $80 \mathrm{~nm}$ polycrystalline & $2 \mathrm{~nm}$ & & $\mathrm{Si}$ & {$[68]$} \\
\hline 14-300 nanocrystalline & $0.18-2.5 \mathrm{~nm}$ & & Glass & [69] \\
\hline 97 nm monocrystalline & 0.82 & & Mica & [70] \\
\hline $10-20 \mathrm{~nm}$ & $0.4 \mathrm{~nm}$ & $\mathrm{Ge}$ & Silica & [73] \\
\hline $5-8.5 \mathrm{~nm}$ & $0.22-0.73 \mathrm{~nm}$ & $\mathrm{Ge}$ & Silica & [74] \\
\hline $15 \mathrm{~nm}$ & $0.6 \mathrm{~nm}$ & $\mathrm{Ge}$ & Silica/Si & [75] \\
\hline $10 \mathrm{~nm}$ & & AZO & & [78] \\
\hline $6 \mathrm{~nm}$ & $0.4 \mathrm{~nm}$ & $\mathrm{Cu}$ & Silicon & [79] \\
\hline $30-280 \mathrm{~nm}$ & $0.65 \mathrm{~nm}$ & & Mica, Si, silica & [80] \\
\hline
\end{tabular}


slightly above 1335 articles in the last 15 years. Meanwhile, the same search, but with silver, gives one order of magnitude larger number of articles, namely almost 15,000 articles, and gold plasmon or metamaterial search almost doubles the latter number, reaching about 26,000 articles.

Owing to its CMOS applicability, the research in depositing thin aluminum films is not confined only to plasmonics and metamaterials. Thus, we present in the following the various methods that could be used for depositing thin Al films.

Initial studies show that evaporated aluminum might form a continuous layer even at thicknesses as low as $4 \mathrm{~nm}$ [81]. Generally, for plasmonic experiments, an aluminium film deposited by an e-beam or thermal evaporation is above $20 \mathrm{~nm}$ in thickness [82]. One certain plasmonic application of an ultrathin Al layer for guiding hybrid long-ranged SPPs was reported in [83]. The thickness of the layer deposited by evaporation was $15 \mathrm{~nm}$. Unfortunately, no any material characterization data of the metal layer structure were provided.

A low-cost chemical way to deposit aluminum is reported in [24]. It is called the solution-dipping process and is based, in fact, on a two-step procedure with first catalytic treatment of the substrate and then immersing it in an aluminum precursor solution. The obtained films have a minimum thickness of ca. $15 \mathrm{~nm}$ and relatively low roughness. As the authors are intending to use these films for electrical interconnects, the films were characterized in terms of the electrical sheet resistance. More studies to show the possibility of using this simple and effective process in plasmonic-related applications are needed.

Dubois et al. [84] reported on the possibility of CVD deposition of Al from precursors through thermal decomposition. The deposition rate was ca. $100 \mathrm{~nm} / \mathrm{min}$, thus, theoretically allowing the possibility of reaching layer thickness below $10 \mathrm{~nm}$. However, there are, to the best of our knowledge, no reports of attempts to minimize the aluminum film thickness.

Aluminum can be also deposited by laser-assisted CVD as in [85]. In this case, the deposition rate of aluminum is comparable to the one of conventional thermal CVD, but further studies are needed to ascertain the possibility of using these films for photonics and especially plasmonics.

Another technique used for depositing thin aluminum films is sputtering. Metals sputter easily; thus, it is not surprising that magnetron sputtering of aluminum is a well-known technique [86-88]. This technique allows for layers of tens of nanometer thickness at least but, up to this date, has not been used in plasmonic research.

Monocrystalline aluminum was successfully deposited on GaAs substrates using the MBE technique [89]. The authors mention thicknesses ranging from 30 to $100 \mathrm{~nm}$.
The main advantage of this technique is the monocrystalline growth; thus, theoretically, lower losses for plasmon propagation can be presumed. One big disadvantage of this deposition is the substrate: GaAs, although having high transmittivity in the IR region, cannot be used in the visible part of the spectrum. To our knowledge, no plasmonic experiments with these films have been performed.

\subsection{Other metals}

Apart from the three metals mentioned, there have been very few attempts to use other metals for plasmonic purposes. This is mainly due to the high losses other metals have or, in the case of alkali metals that might provide lower losses, their instability. Only copper deposition has been more or less addressed due to its CMOS compatibility. Nevertheless, even simple analysis of SPP losses on a metal-dielectric interface showed that copper as a plasmonic materials leads to less losses in the near-infrared range than $\mathrm{Al}$ [90]. In addition, technologically, $\mathrm{Cu}$ is replacing aluminum as the material for interconnects. So far, initial experiments with copper-based structures have been reported [91, 92], but definitely, deposition of thin copper layers is still at the beginning.

Other metals have been addressed rather sporadically. There are some publication about iron [93], nickel [94], and chromium [95] deposition, but no thorough investigation of thin layer deposition of such metals for plasmonics has been performed. This is also due to the theoretically predicted very high losses these metals would have, thus, making them impractical for commercial devices. A very short overview of alternative metals is given in Table 3.

\section{Transparent conductive oxides and conductive nitride films}

Another class of materials that is more and more popular for plasmonic structures is the transparent conductive oxides (TCOs). Although the TCOs have not such a big negative

Table 3: Other metallic thin film parameters on different substrates and using various adhesion layers.

\begin{tabular}{|c|c|c|c|c|c|}
\hline Material & Thickness & RMS & Adhesion layer & Substrate & Ref. \\
\hline Al & $15 \mathrm{~nm}$ & & & Soda lime glass & [24] \\
\hline Al & $4 \mathrm{~nm}$ & & & Glass plates & [81] \\
\hline $\mathrm{Al}$ & $30-100 \mathrm{~nm}$ & $3.6 \mathrm{~nm}$ & & GaAs & [89] \\
\hline $\mathrm{Cu}$ & $15 \mathrm{~nm}$ & & & $\mathrm{TiO}_{2}$ & [92] \\
\hline
\end{tabular}


permittivity as noble metals, their losses are comparable, especially in the IR range, making them a competitive alternative to noble metals. One more advantage is the possibility of tuning their permittivity by design through deciding the dopants or the ratio of different components $[13,96]$, thus constituting an advantage over metals having the fixed permittivity values. By exploring this tuning possibility, plasmonic modulators where the TCOs are the active parts were investigated theoretically [97] and experimentally [98].

\subsection{Indium tin oxide films}

Indium tin oxide (ITO) is the most used TCO nowadays. One of the main advantages of ITO is that its plasma frequency can be tuned through varying the proportions between $\mathrm{SnO}_{2}$ and $\mathrm{In}_{2} \mathrm{O}_{3}[12,96]$ or by the deposition and annealing conditions (see [10] and references therein). Although the ITO plasma frequency is in the near-IR range $[12,17,99,100]$, due to low losses, ITO could be used for plasmonic metamaterials in certain frequency ranges above the plasma frequency [10] and as an active material in plasmonic modulators [101-104] or as infrared antennae [105].

The main technique for depositing ITO is magnetron sputtering, and the properties of the obtained thick films are thoroughly investigated [106-115]. Using this technique, films with thicknesses as low as $10 \mathrm{~nm}$ may be deposited $[98,116]$, although their uniformity was not investigated.

Other methods for depositing ITO include PLD [49] with a reported thickness of $30 \mathrm{~nm}$ (Figure 7) and thermal evaporation $[117,118]$ techniques. In the latter case, the ITO thickness was between 50 and $110 \mathrm{~nm}$.

\subsection{Other conductive oxide films}

Apart from ITO, there are several other TCOs that have been tested as possible plasmonic materials for the IR range. Zinc
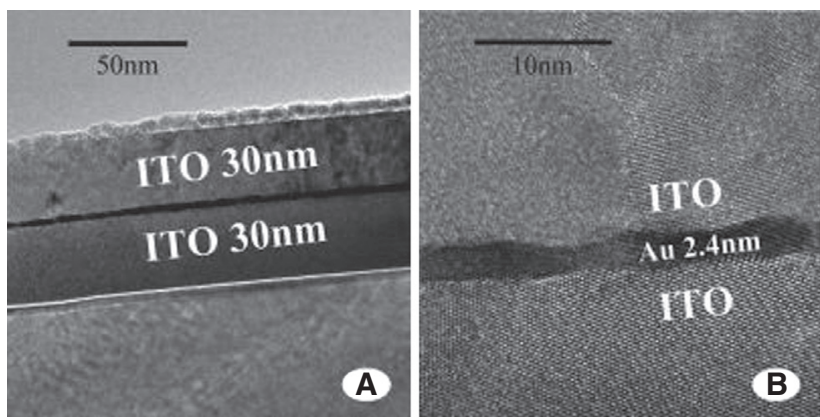

Figure 7: Low roughness 30-nm-thick ITO layers. Reproduced with permission from [49]. oxide-based TCOs are among the ones mainly used. Initial experiments with aluminum doped [78], gallium doped [119], indium doped [120], tin doped [121], or pure [122] zinc oxide have been performed. Apart from the zinc oxidebased TCOs, niobium-doped anatase titanium oxide [123] or indium-doped cadmium oxide [124] were investigated. Still, their properties in the IR range are not fully understood in order to be a weighty alternative to metallic layers.

The deposition technique mainly used for obtaining thin layers are similar to the ones for ITO; thus, magnetron sputtering is the main one [125-127]. Other methods for depositing TCOs, include PLD [123, 128-131], ion beam sputtering [122, 132], and pulsed filtered cathodic arc deposition [124].

\subsection{Interstitial metal nitrides}

Recently, films made of nitrides (titanium nitride, zirconium nitride, tantalum nitride, and hafnium nitride) have gained more prominence in the plasmonics research [133]. Their typical working wavelength range, in order to be used for plasmonic effects, is in the IR. The main appealing properties of nitrides are CMOS compatibility, mechanical hardness, and high-temperature resistance, neutrality toward biological objects and the possibility of fine tuning of parameters by adjusting conditions of deposition. There are plenty of technological approaches to deposit nitrides films. For example, by magnetron sputtering, TiN layers of thicknesses between 30 and $50 \mathrm{~nm}$ can be obtained. Excitation of surface plasmons on such interfaces were reported [134].

\section{Other types of thin films for plasmonics}

\subsection{Highly doped semiconductor films}

Recently, the possibility for growing highly doped semiconductors allowed the plasma frequency of these layers to be in the range of $100 \mathrm{THz}[135,136]$. So far, it has not yet reached the near-IR nor visible range due to the effects of doping compensation appearing at very high doping levels. However, the latest quest for mid-IR plasmonics and photonics makes the highly doped semiconductor as one of the promising candidates to substitute noble metals. Large bandwidth tunability of the plasma frequency in semiconductors by the doping level, for example, between 6 and $16 \mu \mathrm{m}$ [137], also raises expectations for 
semiconductor plasmonics. Nowadays, there are significant efforts toward reaching higher doping levels [138140] thus, pushing plasma frequencies up in the range of hundreds of $\mathrm{THz}$ and further.

The main advantage of these films is the growth methods. Thus, highly doped semiconductors are generally grown using epitaxial methods like molecular beam epitaxy [135, 137], metal-organic chemical vapor deposition [141], or metal-organic vapor phase epitaxy [142, 143]. These techniques allow for atomically smooth layers, greatly reducing the influence of roughness in the devices. On the other hand, epitaxial growth is possible only on lattice-matched substrates. As such, the substrate is generally the same base material but with lower doping levels. In Table 4, we summarize both highly doped semiconductors as well as TCO parameters used for plasmonics.

\subsection{Graphene and other 2D materials}

The ultimate thickness of a material is one monoatomic layer. With monoatomic arrangement of atoms, no any three-dimensional lattice can be constructed. Therefore, until recently, it was thought not to be possible to obtain such materials, but, with the discovery of graphene, a one monolayer of carbon, this limit was achieved. It proclaimed that the era of two-dimensional materials, whose lattices or atom packaging are confined in-plane, had begun. Among a bunch of several exceptional mechanical and electronic properties of graphene, the one which attracted a lot of attention toward graphene plasmonic applications is the possibility of actively doping the monolayer. Thus, light-matter interactions can be accurately

Table 4: Alternative material film parameters on different substrates and using various adhesion layers.

\begin{tabular}{|c|c|c|c|c|c|}
\hline Material & Thickness & RMS & $\begin{array}{l}\text { Adhesion } \\
\text { layer }\end{array}$ & Substrate & Ref. \\
\hline ITO & 30 & $0.612 \mathrm{~nm}$ & & Glass & [49] \\
\hline ITO & 30 & $0.437 \mathrm{~nm}$ & $\mathrm{Au}$ & ITO & [49] \\
\hline AZO & $21.9 \mathrm{~nm}$ & & & Glass & [78] \\
\hline ITO & 10 & & & $\mathrm{Si}_{3} \mathrm{~N}_{4}$ & [98] \\
\hline ITO & 10 & & & Quartz & [116] \\
\hline $\mathrm{ZnO}$ & $20-45$ & & & Glass & [122] \\
\hline $\mathrm{ZnO}$ & 35 & & $\mathrm{Ag}$ & $\mathrm{ZnO}$ & [122] \\
\hline GZO & $25-147 \mathrm{~nm}$ & & & $\mathrm{Al}_{2} \mathrm{O}_{3}$ & [130] \\
\hline $\mathrm{ZnO}$ & $15-50 \mathrm{~nm}$ & & & Glass & [132] \\
\hline $\mathrm{ZnO}$ & $15-50 \mathrm{~nm}$ & & $\mathrm{Cu}$ & $\mathrm{ZnO}$ & [132] \\
\hline $\mathrm{TiN}$ & $30-50 \mathrm{~nm}$ & & & GaAs & [134] \\
\hline GaAsSb & & $0.2395 \mathrm{~nm}$ & & $\ln P$ & [141] \\
\hline InGaAs & $80 \mathrm{~nm}$ & & & AllnAs & [144] \\
\hline AllnAs & $80 \mathrm{~nm}$ & & & InGaAs & [144] \\
\hline
\end{tabular}

controlled through the gating voltage giving the possibility to obtain tunable devices [145-147].

Although there are several ways of obtaining graphene [148], the most used two are through exfoliation from graphite $[149,150]$ and using CVD techniques on $\mathrm{Cu}$ [151] or Ni [152] substrates.

The main advantage of the graphite exfoliation technique is the possibility of obtaining large areas of high-quality monocrystalline graphene on, in principle, whichever substrate. However, the obtained flakes are difficult to position precisely in case of needed alignment, and the flakes sizes are quite random.

On the other hand, CVD techniques have the possibility of wafer-scale manufacturing. The disadvantage lies in the difficulties for obtaining big monocrystalline domains; thus, the graphene response will be highly influenced by the borders of these domains. Techniques for improving the contacts between two domains with different crystallographic orientation joints are currently investigated and might lead to the use of graphene as plasmonic material. Another disadvantage due to the growth method is a substrate. When using CVD techniques, the substrate generally needs to be metallic; thus, further steps have to be made in order to transfer the obtained layer on the desired substrate. These steps will also lead to the introduction of defects in the layer, both physical, through cracks and folds, and chemical, through unwanted doping. Recently, a CVD technique for graphene on silica was developed [153], thus, expanding the substrate range.

Besides graphene, a family of two-dimensional materials with monoatomic arrangement of atoms has potentially emerged. For example, boron nitride, molybdenum bisulfide, and tungsten sulfide [148] are possible candidates to achieve controllable, wafer-scale monoatomic thicknesses. Nevertheless, these types of two-dimensional materials have only recently appeared in the focus of investigation, and their comprehensive optical characterization is yet to be done. It is interesting that twodimensional materials put fabrication challenges to the reverse extreme, when having a monolayer processing more or less established the problem is how to fabricate a few layers thick uniform materials with prescribed sizes.

\section{Conclusions}

The definition of ultra-smooth or ultra-thin layers is subjected to functionality of a concrete device or an operational frequency range. Thus, what can be ultra-smooth in one case can be extremely rough in another. Here, 
we have reviewed the techniques for fabricating layers that can be considered extremely smooth for visible and infra-red radiation thus aiming at the average roughness below $1 \mathrm{~nm}$ and layers thicknesses ideally below $10 \mathrm{~nm}$. Such layers would allow for lower losses in devices that are based on plasmon propagation and localization or metal-dielectric metamaterial performance, thus, helping in pushing plasmonic-based devices toward the market.

Obtaining films with the prescribed thickness and smoothness that nowadays devices require is not, by any means, an easy task. For each specific material, there are several approaches that may lead to extremely smooth, ultra-thin layers; however, there is no ideal universal solution to date. In the above, we have tried to summarize the main types of plasmonic materials and the deposition techniques that allow for ultra-smooth and thin layers to be obtained.

As a word of caution, the losses in plasmonic devices can be minimized only to their theoretical limit. There are already critical works that speculate that even the theoretical limit might not be low enough for market applications [154, 155]. We believe that, although the losses might not reach the desired level for many applications, improving the techniques for depositing ultra-thin layers is a worthwhile task in maturing nanotechnology in itself. A smoother and thinner layer may lead to better performances, thus, allowing for some plasmonic devices and circuit components with specific functionalities to be developed at the level of commercial implementation.

\section{References}

[1] Raether H. Surface Plasmons on Smooth and Rough Surfaces and on Gratings, vol. 111. Springer: Berlin Heidelberg, 1988.

[2] Soref R. The past, present, and future of silicon photonics. IEEE J. Sel. Top. Quantum Electron. 2006, 12, 1678-1687.

[3] Maier SA. Plasmonics: Fundamentals and Applications, Springer: New York, USA, 2007.

[4] Bozhevolnyi SI. Plasmonic Nanoguides and Circuits, Pan Stanford Publishing: Singapore, 2008.

[5] Berini P. Long-range surface plasmon polaritons. Adv. Opt. Photonics 2009, 1, 484-588.

[6] Logeeswaran VJ, Chan M-L, Bayam Y, Saif Islam M, Horsley DA, Li X, Wu W, Wang SY, Williams RS. Ultra-smooth metal surfaces generated by pressure-induced surface deformation of thin metal films. Appl. Phys. A 2007, 87, 187-192.

[7] Kinsey N, Ferrera M, Shalaev VM, Boltasseva A. Examining nanophotonics for integrated hybrid systems: a review of plasmonic interconnects and modulators using traditional and alternative materials [Invited]. J. Opt. Soc. Am. B 2014, 32, 121-142.

[8] Noginov MA, Barnakov YA, Zhu G, Tumkur T, Li H, Narimanov EE. Bulk photonic metamaterial with hyperbolic dispersion. Appl. Phys. Lett. 2009, 94, 151105.
[9] Kidwai O, Zhukovsky SV, Sipe JE. Effective-medium approach to planar multilayer hyperbolic metamaterials: strengths and limitations. Phys. Rev. A 2012, 85, 53842.

[10] West PR, Ishii S, Naik GV, Emani NK, Shalaev VM, Boltasseva A. Searching for better plasmonic materials. Laser Photon. Rev. 2010, 4, 795-808.

[11] Blaber MG, Arnold MD, Ford MJ. A review of the optical properties of alloys and intermetallics for plasmonics. J. Phys. Condens. Matter. 2010, 22, 143201.

[12] Naik GV, Kim J, Boltasseva A. Oxides and nitrides as alternative plasmonic materials in the optical range [Invited]. Opt. Mater. Express 2011, 1, 1090-1099.

[13] Naik GV, Schroeder JL, Ni X, Kildishev AV, Sands TD, Boltasseva A. Titanium nitride as a plasmonic material for visible and nearinfrared wavelengths. Opt. Mater. Express 2012, 2, 478-489.

[14] Frölich A, Wegener M. Spectroscopic characterization of highly doped $\mathrm{ZnO}$ films grown by atomic-layer deposition for three-dimensional infrared metamaterials [Invited]. Opt. Mater. Express 2011, 1, 883-889.

[15] Noginov MA, Gu L, Livenere J, Zhu G, Pradhan AK, Mundle R, Bahoura M, Barnakov YA, Podolskiy VA. Transparent conductive oxides: plasmonic materials for telecom wavelengths. Appl. Phys. Lett. 2011, 99, 21101.

[16] Li D, Ning CZ. All-semiconductor active plasmonic system in mid-infrared wavelengths. Opt. Express 2011, 19, 1459414603.

[17] Naik GV, Boltasseva A. Semiconductors for plasmonics and metamaterials. Phys. status solidi Rapid Res. Lett. 2010, 4, 295-297.

[18] Grigorenko AN, Polini M, Novoselov KS. Graphene plasmonics. Nat. Photonics 2012, 6, 749-758.

[19] Low T, Avouris P. Graphene plasmonics for terahertz to midinfrared applications. ACS Nano 2014; 8, 1086-1101.

[20] Sze SM, Lee M-K. Semiconductor Devices: Physics and Technology, 3rd ed., New York: John Wiley \& Sons, 2012.

[21] May GS, Sze SM. Fundamentals of Semiconductor Fabrication. New York: John Wiley \& Sons, 2007, p. 305.

[22] Malureanu R, Zalkovskij M, Andryieuski A, Lavrinenko AV. Controlled Ag electroless deposition in bulk structures with complex three-dimensional profiles. J. Electrochem. Soc. 2010, 157, K284.

[23] Radke A, Gissibl T, Klotzbücher T, Braun PV, Giessen H. Threedimensional bichiral plasmonic crystals fabricated by direct laser writing and electroless silver plating. Adv. Mater. 2011, 23, 3018-3021.

[24] Lee HM, Choi S-Y, Jung A. Direct deposition of highly conductive aluminum thin film on substrate by solution-dipping process. ACS Appl. Mater. Interfaces 2013, 5, 4581-4585.

[25] Joy DC. Scanning Electron Microscopy, Wiley-VCH Verlag GmbH \& Co. KGaA: Weinheim, 2006.

[26] Eaton P, West P. Atomic Force Microscopy, Oxford University Press: New York, 2010, p. 256.

[27] Williams DB, Carter CB. The Transmission Electron Microscopy, Springer: US, 1996.

[28] Stroscio JA, Kaiser WJ. Scanning Tunelling Microscopy. San Diego: Academic Press, Inc., 1993.

[29] Ullman A. Characterisation of Organic Thin Films. Momentum Press: New York, 2010.

[30] Ichimiya A, Cohen PI. Reflection High Energy Electron Diffraction, Cambridge: Cambridge University Press, 2004. 
[31] Kawata S, Ohtsu M, Irie M. Near-Field Optics and Surface Plasmon Polaritons. Springer-Verlag GMBH: Berlin, 2001.

[32] vom Felde A, Sprösser-Prou J, Fink J. Valence-electron excitations in the alkali metals. Phys. Rev. B 1989; 40, 10181-10193.

[33] Ritchie R. Plasma losses by fast electrons in thin films. Phys. Rev. 1957, 106, 874-881.

[34] Kretschmann E. The determination of the optical constants of metals by excitation of surface plasmons. Eur. Phys. J. A 1971, 241, 313-324.

[35] Giergiel J, Reed CE, Hemminger JC, Ushioda S. Surface plasmon polariton enhancement of Raman scattering in a Kretschmann geometry. J. Phys. Chem. 1988, 92, 5357-5365.

[36] Pollard JD, Sambles JR. The time-dependence of the growth of thin organic liquid layers on a gold surface, studied by surfaceplasmon polariton techniques. Opt. Commun. 1987, 64, 529-533.

[37] Innes RA, Sambles JR. Optical characterisation of gold using surface plasmon-polaritons. J. Phys. F Met. Phys. 1987, 17, 277-287.

[38] Voss R, Laibowitz R, Allessandrini E. Fractal (Scaling) clusters in thin gold films near the percolation threshold. Phys. Rev. Lett. 1982, 49, 1441-1444.

[39] Mahapatro AK, Scott A, Manning A, Janes DB. Gold surface with sub-nm roughness realized by evaporation on a molecular adhesion monolayer. Appl. Phys. Lett. 2006, 88, 151917.

[40] You H, Chiarello R, Kim H, Vandervoort K. X-ray reflectivity and scanning-tunneling-microscope study of kinetic roughening of sputter-deposited gold films during growth. Phys. Rev. Lett. 1993, 70, 2900-2903.

[41] Chiarello RP, You H, Kim HK, Roberts T, Kempwirth RT, Miller D, Gray KE, Vandervoort KG, Trivedi N, Phillpot SR, Zhang QJ, Williams S, Ketterson JB. X-ray reflectivity study on gold films during sputter deposition. Surf. Sci. 1997, 380, 245-257.

[42] Park JW, Pedraza AJ, Allen WR. The interface between sputterdeposited gold thin films and ion-bombarded sapphire substrates. Appl. Surf. Sci. 1996, 103, 39-48.

[43] Varchenya SA, Simanovskis A, Stolyarova SV. Adhesion of thin metallic films to non-metallic substrates. Thin Solid Films 1988, 164, 147-152.

[44] Lee M-L, Sheu J-K, Hu CC. Nonalloyed Cr/Au-based Ohmic contacts to n-GaN. Appl. Phys. Lett. 2007, 91, 182106.

[45] Majni G, Ottaviani G, Prudenziati M. Interdiffusion of thin $\mathrm{Cr}$ and Au films deposited on silicon. Thin Solid Films 1976, 38, 15-19.

[46] Habteyes TG, Dhuey S, Wood E, Gargas D, Cabrini S, Schuck PJ, Alivisatos AP, Leone SR. Metallic adhesion layer induced plasmon damping and molecular linker as a nondamping alternative. ACS Nano 2012, 6, 5702-5709.

[47] Kästle G, Boyen H-G, Koslowski B, Plettl A, Weigl F, Ziemann P. Growth of thin, flat, epitaxial 0 oriented gold films on c-cut sapphire. Surf. Sci. 2002, 498, 168-174.

[48] Leosson K, Ingason AS, Agnarsson B, Kossoy A, Olafsson S, Gather MC. Ultra-thin gold films on transparent polymers. Nanophotonics 2013, 2, 3-11.

[49] Fang X, Mak CL, Dai J, Li K, Ye H, Leung CW. ITO/Au/ITO sandwich structure for near-infrared plasmonics. ACS Appl. Mater. Interfaces 2014, 6, 15743-15752.

[50] Goss CA, Charych DH, Majda M. Application of (3-mercaptopropyl)trimethoxysilane as a molecular adhesive in the fabrication of vapor-deposited gold electrodes on glass substrates. Anal. Chem. 1991, 63, 85-88.
[51] Leandro L, Malureanu R, Rozlosnik N, Lavrinenko A. Ultrathin, ultrasmooth gold layer on dielectrics without the use of additional metallic adhesion layers. ACS Appl. Mater. Interfaces 2015, 7, 5797-5802.

[52] Kossoy A, Simakov D, Olafsson S, Leosson K. Determining surface coverage of ultra-thin gold films from X-ray reflectivity measurements. Thin Solid Films 2013, 536, 50-53.

[53] Kawasaki M, Uchiki H. Sputter deposition of atomically flat $\mathrm{Au}(111)$ and Ag(111) films. Surf. Sci. 1997, 388, L1121-L1125.

[54] Fedotov VA, Uchino T, Ou JY. Low-loss plasmonic metamaterial based on epitaxial gold monocrystal film. Opt. Express 2012, 20, 9545 .

[55] Hugall JT, Finnemore AS, Baumberg JJ, Steiner U, Mahajan S. Solvent-resistant ultraflat gold using liquid glass. Langmuir 2012, 28, 1347-1350.

[56] Hegner M, Wagner P, Semenza G. Ultralarge atomically flat template-stripped Au surfaces for scanning probe microscopy. Surf. Sci. 1993, 291, 39-46.

[57] Piscopiello E, Tapfer L, Antisari M, Paiano P, Prete P, Lovergine N. Formation of epitaxial gold nanoislands on (100) silicon. Phys. Rev. B 2008, 78, 35305.

[58] Huang J-S, Callegari V, Geisler P, Brüning C, Kern J, Prangsma JC, Wu X, Feichtner T, Ziegler J, Weinmann P, Kamp $M$, Forchel A, Biagioni P, Sennhauser U, Hecht B. Atomically flat single-crystalline gold nanostructures for plasmonic nanocircuitry. Nat. Commun. 2010, 1, 150.

[59] Igumenov IK. MO CVD of noble metals. Le J. Phys. IV 1995, 05, C5-489-C5-496.

[60] Okumura M, Nakamura S, Tsubota S, Nakamura T, Azuma M, Haruta M. Chemical vapor deposition of gold on Al203, SiO2, and $\mathrm{TiO} 2$ for the oxidation of $\mathrm{CO}$ and of H2. Catal. Letters 1998 , 51, 53-58.

[61] Gu D, Zhang C, Wu Y-K, Guo LJ. Ultrasmooth and thermally stable silver-based thin films with subnanometer roughness by aluminum doping. ACS Nano 2014, 8, 10343-10351.

[62] Antonello A, Jia B, He Z, Buso D, Perotto G, Brigo L, Brusatin G, Guglielmi M, Gu M, Martucci A. Optimized electroless silver coating for optical and plasmonic applications. Plasmonics 2012, 7, 633-639.

[63] Rill MS, Plet C, Thiel M, Staude I, von Freymann G, Linden S, Wegener M. Photonic metamaterials by direct laser writing and silver chemical vapour deposition. Nat. Mater. 2008, 7, 543-546.

[64] Wu Y, Zhang C, Estakhri NM, Zhao Y, Kim J, Zhang M, Liu X-X, Pribil GK, Alù A, Shih C-K, Li X. Intrinsic optical properties and enhanced plasmonic response of epitaxial silver. Adv. Mater. 2014, 26, 6106-6110.

[65] Smith AR, Chao K-J, Niu Q, Shih C-K. Formation of atomically flat silver films on GaAs with a 'Silver Mean' quasi periodicity. Science 1996, 273, 226-228.

[66] Yu H, Jiang C, Ebert P, Wang X, White J, Niu Q, Zhang Z, Shih C. Quantitative determination of the metastability of flat Ag overlayers on GaAs(110). Phys. Rev. Lett. 2001, 88, 16102.

[67] Lu Y-J, Kim J, Chen H-Y, Wu C, Dabidian N, Sanders CE, Wang C-Y, Lu M-Y, Li B-H, Qiu X, Chang W-H, Chen L-J, Shvets G, Shih C-K, Gwo S. Plasmonic nanolaser using epitaxially grown silver film. Science 2012, 337, 450-453.

[68] Li B-H, Sanders CE, Mcllhargey J, Cheng F, Gu C, Zhang G, Wu K, Kim J, Mousavi SH, Khanikaev AB, Lu Y-J, Gwo S, Shvets G, 
Shih C-K, Qiu X. Contrast between surface plasmon polaritonmediated extraordinary optical transmission behavior in epitaxial and polycrystalline $\mathrm{Ag}$ films in the mid- and far-infrared regimes. Nano Lett. 2012, 12, 6187-6191.

[69] Kapaklis V, Poulopoulos P, Karoutsos V, Manouras T, Politis C. Growth of thin Ag films produced by radio frequency magnetron sputtering. Thin Solid Films 2006, 510, 138-142.

[70] Park JH, Ambwani P, Manno M, Lindquist NC, Nagpal P, Oh S-H, Leighton C, Norris DJ. Single-Crystalline silver films for plasmonics. Adv. Mater. 2012, 24, 3988-3992.

[71] Niskanen A, Hatanpää T, Arstila K, Leskelä M, Ritala M. RadicalEnhanced atomic layer deposition of silver thin films using phosphine-adducted silver carboxylates. Chem. Vap. Depos. 2007, 13, 408-413.

[72] Delgado JM, Orts JM, Rodes A. A comparison between chemical and sputtering methods for preparing thin-film silver electrodes for in situ ATR-SEIRAS studies. Electrochim. Acta 2007, 52, 4605-4613.

[73] Logeeswaran VJ, Kobayashi NP, Islam MS, Wu W, Chaturvedi P, Fang NX, Wang SY, Williams RS. Ultrasmooth silver thin films deposited with a germanium nucleation layer. Nano Lett. 2009, 9, 178-182.

[74] Chen W, Thoreson MD, Ishii S, Kildishev AV, Shalaev VM. Ultrathin ultra-smooth and low-loss silver films on a germanium wetting layer. Opt. Express 2010, 18, 5124-5134.

[75] Logeeswaran VJ, Katzenmeyer A, Islam MS, Kobayashi NP, Wu W, Chaturvedi P, Fang NX, Wang SY, Williams RS. Electrical resistivity \& thermal stability of smooth silver thin film for nanoscale optoelectronic devices. 2008 8th IEEE Conf. Nanotechnol. 2008, 92-94.

[76] Sergeant NP, Hadipour A, Niesen B, Cheyns D, Heremans P, Peumans P, Rand BP. Design of transparent anodes for resonant cavity enhanced light harvesting in organic solar cells. Adv. Mater. 2012, 24, 728-732.

[77] Lajaunie L, Boucher F, Dessapt R, Moreau P. Strong anisotropic influence of local-field effects on the dielectric response of Q-MoO_\{3\}.Phys. Rev. B 2013, 88, 115141.

[78] Guske JT, Brown J, Welsh A, Franzen S. Infrared surface plasmon resonance of AZO-Ag-AZO sandwich thin films. Opt. Express, 2012, 20, 23215-23226.

[79] Formica N, Ghosh DS, Carrilero A, Chen TL, Simpson RE, Pruneri V. Ultrastable and atomically smooth ultrathin silver films grown on a copper seed layer. ACS Appl. Mater. Interfaces 2013, 5, 3048-3053.

[80] Nagpal P, Lindquist NC, Oh S-H, Norris DJ. Ultrasmooth patterned metals for plasmonics and metamaterials. Science 2009, 325, 594-597.

[81] Hass G, Waylonis JE. Optical constants and reflectance and transmittance of evaporated aluminum in the visible and ultraviolet. J. Opt. Soc. Am. 1961, 51, 719-722.

[82] Lahiri B, McMeekin SG, Khokhar AZ, De La Rue RM, Johnson NP. Magnetic response of split ring resonators (SRRs) at visible frequencies. Opt. Express 2010, 18, 3210-3218.

[83] Zektzer R, Desiatov B, Mazurski N, Bozhevolnyi SI, Levy U. Experimental demonstration of CMOS-compatible long-range dielectric-loaded surface plasmon-polariton waveguides (LRDLSPPWs). Opt. Express 2014, 22, 22009-22017.

[84] Dubois LH, Zegarski BR, Gross ME, Nuzzo RG. Aluminum thin film growth by the thermal decomposition of triethylamine alane. Surf. Sci. 1991, 244, 89-95.
[85] Solanki R, Ritchie WH, Collins GJ. Photodeposition of aluminum oxide and aluminum thin films. Appl. Phys. Lett. 1983, 43, 454-456.

[86] McLeod PS, Hartsough LD. High-rate sputtering of aluminum for metallization of integrated circuits. J. Vac. Sci. Technol. 1977, 14, 263.

[87] Hartsough LD, McLeod PS. High-rate sputtering of enhanced aluminum mirrors. J. Vac. Sci. Technol. 1977, 14, 123.

[88] Arnell R, Bates R. The deposition of highly supersaturated metastable aluminium-magnesium alloys by unbalanced magnetron sputtering from composite targets. Vacuum 1992, 43, 105-109.

[89] Lin S-W, Wu J-Y, Lin S-D, Lo M-C, Lin M-H, Liang C-T. Characterization of single-crystalline aluminum thin film on (100) GaAs substrate. Jpn. J. Appl. Phys. 2013, 52, 45801.

[90] Emboras A, Najar A, Nambiar S, Grosse P, Augendre E, Leroux C, de Salvo B, de Lamaestre RE. MNOS stack for reliable, low optical loss, Cu based CMOS plasmonic devices. Opt. Express 2012, 20, 13612-13621.

[91] Volpati D, Spada ER, Plá Cid CC, Sartorelli ML, Aroca RF, Constantino CJL. Exploring copper nanostructures as highly uniform and reproducible substrates for plasmon-enhanced fluorescence. Analyst 2015, 140, 476-482.

[92] Liu Z, Liu G, Liu X, Huang S, Liu M, Fu G, Gao H. Continuous copper film structures with broadband optical transparency. Mater. Lett. 2015, 139, 12-14.

[93] Chong X, Abboud J, Zhang Z. Plasmonics resonance enhanced active photothermal effects of aluminum and iron nanoparticles. J. Nanosci. Nanotechnol. 2015, 15, 2234-2240.

[94] Hamidi SM, Sobhani A, Aftabi A, Najafi M. Optical and magneto-optical properties of aligned $\mathrm{Ni}$ nanowires embedded in polydimethylsiloxane. J. Magn. Magn. Mater. 2015, 374, 139-143.

[95] Quang NK, Miyauchi Y, Mizutani G, Charlton MD, Chen R, Boden S, Rutt H. Optical second harmonic generation of V-shaped chromium nanoholes-dependence on the structure parameters of the nanoholes. Surf. Interface Anal. 2014, 46, 1240-1244.

[96] Mishra SK, Gupta BD. Surface plasmon resonance-based fiber-optic hydrogen sas sensor utilizing indium-tin oxide (ITO) thin films. Plasmonics 2012, 7, 627-632.

[97] Babicheva VE, Kinsey N, Naik GV, Ferrera M, Lavrinenko AV, Shalaev VM, Boltasseva A. Towards CMOS-compatible nanophotonics: ultra-compact modulators using alternative plasmonic materials. Opt. Express 2013, 21, 27326-27337.

[98] Melikyan A, Lindenmann N, Walheim S, Leufke PM, Ulrich S, Ye J, Vincze P, Hahn H, Schimmel T, Koos C, Freude W, Leuthold J. Surface plasmon polariton absorption modulator. Opt. Express 2011, 19, 8855-8869.

[99] Naik GV, Shalaev VM, Boltasseva A. Semiconductor plasmonic metamaterials for near-infrared and telecommunication wavelength. Proc. SPIE 2010, 7754, 77540M-77540M-5.

[100] Naik GV, Boltasseva A. A comparative study of semiconductorbased plasmonic metamaterials. Metamaterials 2011, 5, 1-7.

[101] Babicheva VE, Lavrinenko AV. Plasmonic modulator optimized by patterning of active layer and tuning permittivity. Opt. Commun. 2012, 285, 5500-5507.

[102] Babicheva VE, Malureanu R, Lavrinenko AV. Plasmonic finitethickness metal-semiconductor-metal waveguide as ultracompact modulator. Photonics Nanostructures Fundam. Appl. 2013, 11, 323-334. 
[103] Shi K, Haque RR, Zhao B, Zhao R, Lu Z. Broadband electrooptical modulator based on transparent conducting oxide. Opt. Lett. 2014, 39, 4978-4981.

[104] Zhu S, Lo GQ, Kwong DL. Design of an ultra-compact electroabsorption modulator comprised of a deposited TiN/ $/ \mathrm{HFO}_{2} /$ ITO/Cu stack for CMOS backend integration. Opt. Express 2014, 22, 17930-17947.

[105] Abb M, Wang Y, Papasimakis N, de Groot CH, Muskens OL. Surface-enhanced infrared spectroscopy using metal oxide plasmonic antenna arrays. Nano Lett. 2014, 14, 346-352.

[106] Terzini E, Thilakan P, Minarini C. Properties of ITO thin films deposited by RF magnetron sputtering at elevated substrate temperature. Mater. Sci. Eng. B 2000, 77, 110-114.

[107] Thilakan P, Minarini C, Loreti S, Terzini E. Investigations on the crystallisation properties of RF magnetron sputtered indium tin oxide thin films. Thin Solid Films 2001, 388, 34-40.

[108] Hu Y, Diao X, Wang C, Hao W, Wang T. Effects of heat treatment on properties of ITO films prepared by rf magnetron sputtering. Vacuum 2004, 75, 183-188.

[109] Meng L, dos Santos M. Properties of indium tin oxide films prepared by $\mathrm{rf}$ reactive magnetron sputtering at different substrate temperature. Thin Solid Films 1998, 322, 56-62.

[110] Mergel D, Schenkel M, Ghebre M, Sulkowski M. Structural and electrical properties of In203:Sn films prepared by radiofrequency sputtering. Thin Solid Films 2001, 392, 91-97.

[111] Qiao Z, Latz R, Mergel D. Thickness dependence of In203:Sn film growth. Thin Solid Films 2004, 466, 250-258.

[112] Qiao Z, Mergel D. Comparison of radio-frequency and directcurrent magnetron sputtered thin In203:Sn films. Thin Solid Films 2005, 484, 146-153.

[113] Khosroabadi AA, Gangopadhyay P, Duong B, Thomas ], Sigdel AK, Berry JJ, GennettT, Peyghambarian N, Norwood RA. Fabrication, electrical and optical properties of silver, indium tin oxide (ITO), and indium zinc oxide (IZO) nanostructure arrays. Phys. status solidi 2013, 210, 831-838.

[114] Guillén C, Herrero J. Transparent conductive ITO/Ag/ITO multilayer electrodes deposited by sputtering at room temperature. Opt. Commun. 2009, 282, 574-578.

[115] Chuang S-H, Tsung C-S, Chen C-H, Ou S-L, Horng R-H, Lin C-Y, Wuu D-S. Transparent conductive oxide films embedded with plasmonic nanostructure for light-emitting diode applications. ACS Appl. Mater. Interfaces 2015, 7, 2546-2553.

[116] Pincella F, Isozaki K, Miki K. A visible light-driven plasmonic photocatalyst. Light Sci. Appl. 2014, 3, e133.

[117] Vaishnav VS, Patel PD, Patel NG. Preparation and characterization of indium tin oxide thin films for their application as gas sensors. Thin Solid Films 2005, 487, 277-282.

[118] Mishra SK, Gupta BD. Surface plasmon resonance based fiber optic pH sensor utilizing Ag/ITO/Al/hydrogel layers. Analyst 2013, 138, 2640-2646.

[119] Kim J, Naik GV, Gavrilenko AV, Dondapati K, Gavrilenko VI, Prokes SM, Glembocki OJ, Shalaev VM, Boltasseva A. Optical properties of gallium-doped zinc oxide - a low-loss plasmonic material: first-principles theory and experiment. Phys. Rev. $X$ 2013, 3, 41037.

[120] Craciun V, Martin C, Socol G, Tanner D, Swart HC, Becherescu N, Craciun D. Optical properties of amorphous indium zinc oxide thin films synthesized by pulsed laser deposition. Appl. Surf. Sci. 2014, 306, 52-55.
[121] Winkler T, Schmidt H, Flügge H, Nikolayzik F, Baumann I, Schmale S, Johannes H-H, Rabe T, Hamwi S, Riedl T, Kowalsky W. Realization of ultrathin silver layers in highly conductive and transparent zinc tin oxide/silver/zinc tin oxide multilayer electrodes deposited at room temperature for transparent organic devices. Thin Solid Films 2012, 520, 4669-4673.

[122] El Hajj A, Lucas B, Chakaroun M, Antony R, Ratier B, Aldissi M. Optimization of $\mathrm{ZnO} / \mathrm{Ag} / \mathrm{ZnO}$ multilayer electrodes obtained by lon beam sputtering for optoelectronic devices. Thin Solid Films 2012, 520, 4666-4668.

[123] Su W, Song K, Huo D, Li B. Analysis of correlation between electrical and infrared optical properties of anatase $\mathrm{Nb}$ doped TiO2 films. Curr. Appl. Phys. 1213, 13, 556-561.

[124] Mendelsberg RJ, Zhu Y, Anders A. Determining the nonparabolicity factor of the $\mathrm{CdO}$ conduction band using indium doping and the Drude theory. J. Phys. D. Appl. Phys. 2012, 45, 425302.

[125] Lai K-C, Tsai F-J, Wang J-H, Yeh C-H, Houng M-P. Wet-etch texturing of ZnO:Ga back layer on superstrate-type microcrystalline silicon solar cells. Sol. Energy Mater. Sol. Cells 2011, 95, 1583-1586.

[126] Kim Y-H, Kim D-W, Murakami R-I, Zhang D, Yoon S-W, Park S-H, Moon K-M. The study of transmittance and conductivity by Top $\mathrm{ZnO}$ thickness in $\mathrm{ZnO} / \mathrm{Ag} / \mathrm{ZnO}$ transparent conducting oxide films. Adv. Sci. Lett. 2011, 4, 1570-1573.

[127] Losego MD, Efremenko AY, Rhodes CL, Cerruti MG, Franzen S, Maria J-P. Conductive oxide thin films: model systems for understanding and controlling surface plasmon resonance. J. Appl. Phys. 2009, 106, 24903.

[128] Venugopal N, Kaur G, Mitra A. Plasmonics effect of Ag nanoislands covered n-Al:ZnO/p-Si heterostructure. Appl. Surf. Sci. 2014, 320, 30-42.

[129] Cleary JW, Nader Esfahani N, Vangala S, Guo J, Hendrickson JR, Leedy KD, Look DC. Mid-infrared extraordinary transmission through Ga-doped ZnO films with 2D hole arrays. Proc. SPIE 2014, 8987, 898704.

[130] Allen MS, Allen JW, Wenner BR, Look DC, Leedy KD. Application of highly conductive $\mathrm{ZnO}$ to the excitation of long-range plasmons in symmetric hybrid waveguides. Opt. Eng. 2013, $52,64603$.

[131] Look DC, Droubay TC, Chambers SA. Stable highly conductive $\mathrm{ZnO}$ via reduction of $\mathrm{Zn}$ vacancies. Appl. Phys. Lett. 2012, 101, 102101.

[132] El Hajj A, Lucas B, Barbot A, Antony R, Ratier B, Aldissi M. Organic solar cells using a $\mathrm{ZnO} / \mathrm{Cu} / \mathrm{ZnO}$ anode deposited by ion beam sputtering at room temperature for flexible devices. J. Nanosci. Nanotechnol. 2013, 13, 5227-5232.

[133] Naik GV, Shalaev VM, Boltasseva A. Alternative plasmonic materials: beyond gold and silver. Adv. Mater. 2013, 25, 3264-3294.

[134] Steinmüller-Nethl D, Kovacs R, Gornik E, Rödhammer P. Excitation of surface plasmons on titanium nitride films: determination of the dielectric function. Thin Solid Films 1994, 237, 277-281.

[135] Felts JR, Law S, Roberts CM, Podolskiy V, Wasserman DM, King WP. Near-field infrared absorption of plasmonic semiconductor microparticles studied using atomic force microscope infrared spectroscopy. Appl. Phys. Lett. 2013, 102, 152110.

[136] Ginn JC, Jarecki RL, Shaner EA, Davids PS. Infrared plasmons on heavily-doped silicon. J. Appl. Phys. 2011, 110, 43110. 
[137] Law S, Adams DC, Taylor AM, Wasserman D. Mid-infrared designer metals. Opt. Express 2012, 20, 12155.

[138] Law S, Podolskiy V, Wasserman D. Towards nano-scale photonics with micro-scale photons: the opportunities and challenges of mid-infrared plasmonics. Nanophotonics 2013, 2, 103-130.

[139] Law S, Yu L, Wasserman D. Epitaxial growth of engineered metals for mid-infrared plasmonics. J. Vac. Sci. Technol. B Microelectron. Nanom. Struct. 2013, 31, 3C121.

[140] Hsu S-W, Bryks W, Tao AR. Effects of carrier density and shape on the localized surface plasmon resonances of $\mathrm{Cu} 2-\mathrm{x} \mathrm{S}$ nanodisks. Chem. Mater. 2012, 24, 3765-3771.

[141] Yokoyama H, Hoshi T. P-type conductivity control of Si-doped GaAsSb layers grown by metalorganic chemical vapor deposition. Jpn. J. Appl. Phys. 2015, 54, 15506.

[142] Metaferia W, Kataria H, Sun Y-T, Lourdudoss S. Growth of InP directly on Si by corrugated epitaxial lateral overgrowth. J. Phys. D. Appl. Phys. 2015, 48, 45102.

[143] Ishizaka F, Hiraya Y, Tomioka K, Fukui T. Growth of wurtzite $\mathrm{GaP}$ in $\mathrm{InP} / \mathrm{GaP}$ core-shell nanowires by selective-area MOVPE. J. Cryst. Growth 2015, 411, 71-75.

[144] Hoffman AJ, Alekseyev L, Howard SS, Franz KJ, Wasserman D, Podolskiy VA, Narimanov EE, Sivco DL, Gmachl C. Negative refraction in semiconductor metamaterials. Nat. Mater. 2007, 6, 946-950.

[145] Avouris P, Xia F. Graphene applications in electronics and photonics. MRS Bull. 2012, 37, 1225-1234.

[146] García de Abajo FJ. Applied physics. Graphene nanophotonics. Science 2013, 339, 917-918.
[147] Koppens FHL, Chang DE, García de Abajo FJ. Graphene plasmonics: a platform for strong light-matter interactions. Nano Lett. 2011, 11, 3370-3377.

[148] Chen X, Boulos RA, Eggers PK, Raston CL. p-Phosphonic acid calix[8]arene assisted exfoliation and stabilization of 2D materials in water. Chem. Commun. (Camb). 2012, 48, 11407-11409.

[149] Novoselov KS, Geim AK, Morozov SV, Jiang D, Zhang Y, Dubonos SV, Grigorieva IV, Firsov AA. Electric field effect in atomically thin carbon films. Science 2004, 306, 666-669.

[150] Novoselov KS, Jiang D, Schedin F, Booth TJ, Khotkevich VV, Morozov SV, Geim AK. Two-dimensional atomic crystals. Proc. Natl. Acad. Sci. USA 2005, 102, 10451-10453.

[151] Wang Y, Zheng Y, Xu X, Dubuisson E, Bao Q, Lu J, Loh KP. Electrochemical delamination of CVD-grown graphene film: toward the recyclable use of copper catalyst. ACS Nano 2011, 5, 9927-9933.

[152] Obraztsov AN. Chemical vapour deposition: making graphene on a large scale. Nat. Nanotechnol. 2009, 4, 212-213.

[153] Sun J, Lindvall N, Cole MT, Wang T, Booth TJ, Bøggild P, Teo KBK, Liu J, Yurgens A. Controllable chemical vapor deposition of large area uniform nanocrystalline graphene directly on silicon dioxide. J. Appl. Phys. 2012, 111, 44103.

[154] Khurgin JB, Sun G. In search of the elusive lossless metal. Appl. Phys. Lett. 2010, 96, 181102.

[155] Khurgin JB. How to deal with the loss in plasmonics and metamaterials. Nat. Nanotechnol. 2015, 10, 2-6. 